\title{
Zur autologen Transfusion zwischenzeitlich tiefgefrorenen Blutes im Kindesalter
}

\section{Autologous Transfusion of Deep Frozen Homologous Blood in Infancy}

\author{
St. Kellnar, E. Hansen, U. Hofmann', M. Heim, St. Horst ${ }^{2}$ \\ ${ }^{1}$ Kinderchirurgische Klinik (Direktor: Prof. Dr. W. Ch. Hecker) im Dr. von Haunerschen Kinderspital der Universität München und dem \\ ${ }^{2}$ Transfusionszentrum (Leiter: Prof. Dr. W. Mempel) im Klinikum Großhadern der Universität München
}

Eingegangen am 29. Mai 1986

\section{Zusammenfassung}

Es wird über 71 Kinder und Jugendliche im Alter zwischen 2 und 17 Jahren berichtet, bei denen Brustwandkorrekturen durchgeführt wurden, nachdem ihnen 3 bis 4 Wochen präoperativ 10 bis $15 \%$ des Blutvolumens entnommen war, so daß es nach zwischenzeitlicher Tiefgefrierung zur Retransfusion intraoperativ zur Verfügung stand. Durch diese Maßnahme ließ sich der Anteil der Patienten, die bei Brustwandkorrekturen Fremdblut benötigten, von 18,6\% (vor Einführung der autologen Transfusion) auf 4,2\% senken.

Schlüsselwörter: Autologe Transfusion - Tiefkühlkonservierung von Erythrozyten - Gefrorenes Frischplasma

\section{Summary}

In the report we describe a method of reducing the number of transfusions of heterologous blood in patients with a surgical correction of chest walls. In 71 children between 2 and 17 years of age, $10-15 \%$ of total blood volume were withdrawn 3-4 weeks before the operation, deep frozen and thawed at the appropriate time to be ready for retransfusion. This arrangement reduced the need of heterologous blood significantly from $18.6 \%$ (prior to the introduction of the method) to $4.2 \%$.

Key words: Autologous transfusion - Deep frozen erythrocytes - Fresh frozen plasma

\section{Einleitung}

Präoperative Blutentnahme zur intraoperativen autologen Retransfusion wurde erstmals von Grant (7) beschrieben und in der Folgezeit bei größeren elektiven operativen Eingriffen immer wieder angewandt $(1,4,5,10)$. Die relativ einfach durchführbare Methode, die die Risiken von Infektionsübertragung, Unverträglichkeitsreaktionen und Antikörperbildung bei Fremdblutübertragungen (sog. homologe Transfusion) umgeht, war bisher aufgrund der begrenzten Haltbarkeit von Blutkonserven (bei $+4^{\circ} \mathrm{C}$ maximale Lagerungszeit: 4 Wochen) in ihrer Einsatzmöglichkeit beschränkt. Durch das in den letzten Jahren entwickelte Einfrierverfahren im Tief- temperaturbereich wurde eine Konservierungszeit über mehrere Jahre möglich. Dies gewährleistet neben einer freien Terminwahl zur Blutentnahme die problemlose Entnahme mehrerer Konserven bei dem selben Patienten in ausreichenden Zeitintervallen, um auch bei vorhersehbaren stärkeren Blutverlusten Fremdblutgaben umgehen zu können. Bisher liegen nur wenige Erfahrungen über den Einsatz der autologen Transfusion bei Kindern und Kleinkindern vor $(4,6)$.

\section{Probandenauswahl und Methode}

Dieser Bericht stützt sich auf eine Studie über 71 Patienten im Alter zwischen 2 und 17 Jahren (Durchschnitsalter: 10,2 Jahre) mit ausgeprägter Kiel- oder Trichterbrust. Vor operativer Brustwandkorrektur wurden ihnen einmalig zwischen 10 und $15 \%$ des jeweiligen Blutvolumens entnommen. Eine intravasale Volumenreduktion dieser Menge in einem Zeitraum von länger als 5 Minuten wird von Kindern und Jugendlichen problemlos toleriert (6). Entsprechend einer vorausgegangenen retrospektiven Untersuchung von 263 Patienten, bei denen im Zeitraum von 1978 bis 1984 in unserer Klinik Brustwandkorrekturen durchgeführt wurden, stellte sich heraus, daß bei 49 Patienten (18,6\% des Gesamtkollektives) intraoperativ Fremdblut transfundiert werden mußte. Bei 45 Patienten war die Gabe einer Vollblutkonserve bzw. eines Erythrozytenkonzentrates ausreichend, bei 4 Patienten mußten aufgrund stärkeren intraoperativen Blutverlustes jeweils 2 Vollblutkonserven transfundiert werden. So entschlossen wir uns zu einer einmaligen präoperativen Blutentnahme.

Der eigentlichen Blutentnahme gehen folgende Maßnahmen voraus:

1. Die Entnahmesysteme werden aufgrund unterschiedlicher Blutvolumina modifiziert: Bei größeren Kindern und Jugendlichen mit einem Körpergewicht von über $45 \mathrm{~kg}$ kann die in das geschlossene Beutelsystem eingeschweißte Punktionsnadel benutzt werden. Bei kleineren Kindern adaptieren wir unter „laminar air flow-Bedingungen" einen Dreiwegehahn an das Beutelsystem und konnektieren mit einem „Butterfly“ (19 G) und einer Perfusorspritze (Abb. 1). Vor Entnahme kann dadurch das Volumen der Stabilisatorlösung (ACD) an das Volumen des zu entnehmenden Blutes in dem notwendigen Verhältnis von $1: 7$ angepaßt werden. Während der Entnahme ist außerdem durch primäre Aspiration des Blutes in die Perfusorspritze mit anschließender Einleitung in das Beutelsystem eine exakte Volumenkontrolle möglich.

2. Ein Lokalanästhetikum wird paravenös infiltrierend am Punktionsort appliziert. 
Abb. 1 Für Kleinkinder modifiziertes Entnahmesystem

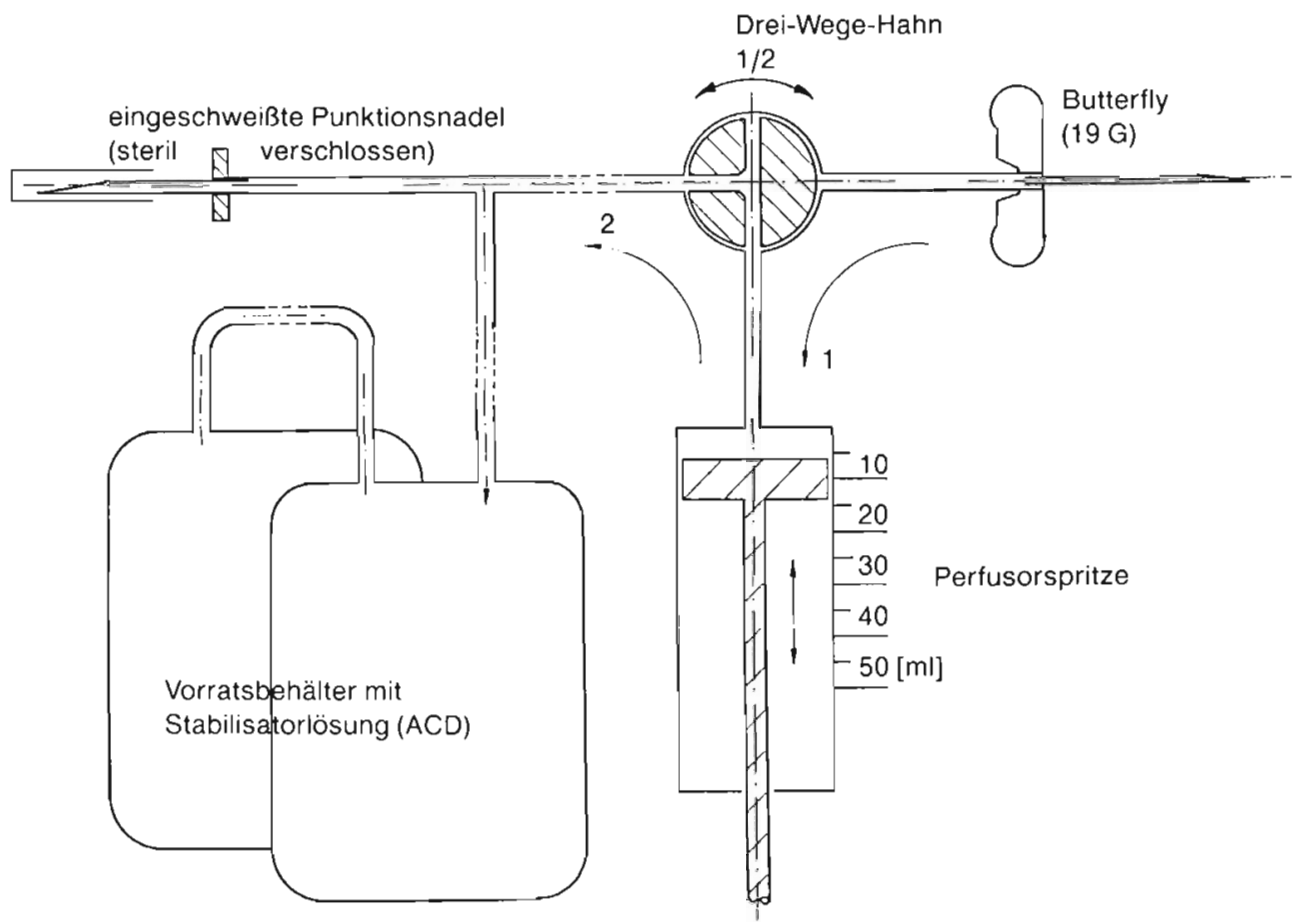

3. Zur Vermeidung hypovolämischer Reaktionen legen wir zusätzlich eine Infusion mit Glucose-Ringer-Lösung an.

Unmittelbar nach Entnahme wird die Eigenblutkonserve in das Transfusionszentrum im KJinikum Großhadern befördert, wo sie nach längstens 3 Stunden durch Zentrifugation in Plasma und Erythrozytensediment aufgetrennt wird. Das Plasma wird bei minus $40^{\circ} \mathrm{C}$ konserviert, das mit $18 \%$ Glycerin versetzte Erylhrozytensediment wird nach Einfrieren bei minus $196^{\circ} \mathrm{C}$ in der Dampfphase des flüssigen Stickstoffs gelagert. Am Operationstag werden die Erythrozytenkonzentrate im Wasserbad nach Auswaschung des Glycerins aufgetaut und mit dem ebenfalls aufgetauten autologen Plasma in die Kinderchirurgische Klinik transportiert.

\section{Ergebnisse}

Die Volumina der autologen Blutkonserven betrugen zwischen $100 \mathrm{ml}$ (2jähriger Junge mit einem Körpergewicht von $9,5 \mathrm{~kg}$ ) und $450 \mathrm{ml}$ (alle Patienten mit einem Körpergewicht über $45 \mathrm{~kg}$ ).

Die Komplikationen bei Entnahme waren gering (Tab. I). Bei 5 Patienten kam es zu vasovagalen Reaktionen: Bei 2 Patienten wurden Schweißausbrüche beobachtet, bei weiteren 2 Patienten trat auffallende Gesichtsblässe auf, und ein 10jähriges Mädchen klagte über Übelkeit und erbrach einmalig. Bei Manifestation eines dieser Symptome stoppten wir sofort die Blutentnahme. Bis auf einen 5jährigen Jungen, bei dem wir aufgrund seiner erheblichen emotionalen Streßsituation die Entnahme auf einen zweiten Termin vertagen mußten,

Tabelle I Komplikationen während und unmittelbar nach der Blutentnahme bei 71 Kindern

Vasovagale Reaktionen:

Übelkeit und Erbrechen

SchweiBausbruch

Auffallende Blässe

Erhebl. emotionaler Streß: konnte jede „Eigenblutspende“ ohne besondere Beeinträchtigung durchgeführt werden. Komplikationen intra- oder postoperativ nach Retransfusion traten nicht auf.

Die Werte der Hämoglobinkonzentrationen vor Entnahme lagen bei durchschnittlich $12,5 \mathrm{~g} \%$. Unmittelbar präoperativ - also ca. 3 Wochen nach Entnahme - war ein Anstieg des durchschnittlichen Hämoglobinwertes auf $13,6 \mathrm{~g} \% \mathrm{zu}$ verzeichnen. Diese Überkompensation ist durch die Stimulation der Erythropoese aufgrund des vorausgegangenen Blutverlustes erklärbar $(3,8)$. Untersucht man die Hämoglobinkonzentrationen unmittelbar präoperativ und am ersten postoperativen Tag, so zeigt sich, daß bei $7 \%$ der Patienten ein Hb-Abfall von $>4 \mathrm{~g} \%$ vorliegt. Vor Einführung der autologen Transfusion (retrospektive Studie aus den Jahren 1977 bis 1984) wurde eine Hb-Differenz von $>4 \mathrm{~g} \%$ in dem entsprechenden Zeitraum bei immerhin $38 \%$ (100/263) ermittelt.

Aufgrund stärkeren intraoperativen Blutverlustes mußte bei 3 Patienten zusätzlich zur obligat retransfundierten autologen Blutkonserve jeweils ein homologes Erythrozytenkonzentrat transfundiert werden. Die Häufigkeitsrate von Fremdblutgaben bei Brustwandkorrekturen sank durch den Einsatz der autologen Bluttransfusion somit von vormals $18,6 \%(49 / 263)$ auf nunmehr 4,2\%(3/71).

\section{Diskussion}

In Abwägung der Vor- und Nachteile (Tab. II) stellt sich die Methode entsprechend unserer Studie als praktikable und wirksame Maßnahme dar, die Risiken von Fremdblutgaben erheblich zu verringern. Neben der Vermeidung von Infektionsübertragungen ist bei Kindern, bei denen aufgrund ihrer Lebenserwartung die Wahrscheinlichkeit von weiteren Bluttransfusionen höher ist als bei Erwachsenen, das Ausbleiben von Antikörperbildung besonders hervorzuheben.

Bei jüngeren Kindern besteht die Gefahr einer psychischen 
Tabe/le // Vor- und Nachteile der autologen Bluttransfusion nach Tiefkühlkonservierung

\section{Vorteile:}

1) Lagerung über Jahre möglich

2) Transfusion auch bei seltenen Blutgruppen gewährleistet

3) Annähernde Frischblutqualität von aufgetauten Erythrozyten und Plasma

4) Vermeidung von Antikörperbildung durch homologe Blutbestandteile

5) Verminderung des Risikos der Infektionsübertragung

\section{Nachteile:}

1) Hohe Investitionskosten

2) Hoher organisatorischer Aufwand

3) Maximale Verwendungsdauer der aufgetauten Erythrozyten: 24 Stunden

4) Beim Kleinkind evtl. traumatisierende Entnahmeprozedur

Traumatisierung durch die Entnahmeprozedur. Deswegen spielt die Kooperation der Eltern eine wesentliche Rolle. Ausnahmslos waren alle Eltern durch ihr beruhigendes Einwirken auf das Kind an der erfolgreichen Blutentnahme beteiligt. Eine besondere Motivation bestand durch die immer wieder von den Eltern erwähnte Furcht vor einer möglichen AIDS-Infektion.

Will man auch jene Fremdblutgaben einsparen, die bei 3 Patienten zusätzlich zur autologen Blutkonserve transfundiert werden mußten, stehen 3 Möglichkeiten zur Diskussion:

1. Die präoperative Abnahme mehrerer Blutkonserven nach bestimmten Zeitintervallen. Die in unserer Untersuchung relativ niedrige Rate der Patienten, die mehr als eine Eigenblutgabe benötigten (4,2\% aller Probanden), rechtfertigt unserer Meinung nach nicht mehr als eine präoperative Blutentnahme, vor allem wegen der psychischen Belastung besonders jüngerer Kinder. Eventuell könnte man bei Patienten, die älter als 14 Jahre sind ( 2 der 3 Patienten, die zusätzlich Fremdblut benötigten, lagen in dieser Altersklasse) routinemäßig eine zweite Blutentnahme einplanen.

2. Die intraoperative Retransfusion des aus dem Operationsfeld abgesaugten und wieder aufgearbeiteten Blutes wird in unserer Klinik nicht durchgeführt, da bei kinderchirurgischen Eingriffen der Anwendungsbereich zu sehr eingeengt ist.

3. Die normovolämische akute präoperative Hämodilution, d. h. die präoperative Eigenblutspende mit Volumenersatz durch Glucose-Ringer-Lösung bis zu einem Hämatokritwert von $25 \%$ wird von jüngeren Patienten ohne Herz-, Kreislauf- oder hämatologische Erkrankungen problemlos vertragen $(2,4,9)$. Das konservierte autologe Blut kann dann je nach Bedarf intra- oder postoperativ retransfundiert werden. Diese Methode wird zur Zeit in unserer Klinik erprobt.

\section{Schlußfolgerung}

Nach unseren Erkenntnissen sollte bei allen größeren elektiven Eingriffen, die erfahrungsgemäß mit größeren Blutverlusten einhergehen und den Einsatz von Fremdblut notwendig machen - die Brustwandkorrektur wird hier nur als Beispiel einer Indikationsstellung vorgestellt -, die präoperative Blutentnahme zur intraoperativen Retransfusion nach zwischen. zeitlicher Tiefgefrierung eingesetzt werden, um die Risiken einer Fremdblutgabe zu vermeiden.

\section{Literatur}

(1) Boerema I, Fierstra A: Praeoperatieve bloedverdunning. Nederlandsch Tijdschr Geneesk 99: 69-73 (1955).

(2) Brzica St, Pineda AA, Taswell

HF: Autologous blood transfusion. Mayo Clin Proc 51: 733-737 (1976). (3) Buerger E: Hämodilution. Klin Transfusionsmed Ed Medizin. Florida, Basel 1982.

(4) Cowell HR, Swickard JW: Autotransfusion in Children's Orthopaedics. J Bone Joint Surg 12: 908-912 (1974).

(5) Fantus B: The therapy of the cook county Hospital. Blood Preservation. J Am Assn 109: 128-131 (1937).

(6) Gilcher RO, Belcher L: Predeposit Programs. Autologous Transfusion. Presented by the Committee on Technical Workshops. American

\section{Für die Verfasser:}

Dr. med. St. Kellnar,

Kinderchirurgische Klinik im

Dr. von Haunerschen Kinderspital der Universitäl München,

Lindwurmstr, 4 ,

8000 München 2
Association of Blood Banks. Arlington, Virginia 1983

(7) Granı FC: Autotransfusion. Ann Surg 24: 253-254 (1921)

(8) Kubaneck B: Physiologie des Blutes. In: Ahnefeld FW, Bergmann H, Burri C, Dick W, Halmágyi M, Hossli G, Rügheimer E: Therapie mit Blutkomponenten. Klin Anästhesiologie und Intensivtherapie.

Band 21. Berlin, Heidelberg, New York: Springer Verlag 1980.

(9) Scsencsitz ThA, Flynn JC: Intraoperative bloodsalvage in spinal deformity surgery in children. I Florida MA 66: 3I-34 (1979).

(10) Turner RS: Autologous Blood for Surgical Autotransfusions. Proceedings of The American Academy of Orthopaedic Surgeons. J Bone Joint Surg 834: 50-A (1968). + 